\title{
THE LOCATION OF THE ZEROS OF POLYNOMIALS WITH COMPLEX COEFFICIENTS
}

\section{EVELYN FRANK}

1. Introduction. In a recent paper On the zeros of polynomials with complex coefficients [2],1 we gave an algorithm for determining the number of zeros in any half-plane. We here extend these methods to the actual computation of the zeros by successive approximation. The computation involves only the repetitive processes of synthetic division and cross-multiplication, and computation with complex numbers has been eliminated. This method is therefore especially suitable where the computing is to be done by a clerical staff with the use of ordinary calculating machines.

While relatively few methods for finding the zeros of complex polynomials have been given, there are many methods for real polynomials. Runge [12], in 1898, made a comprehensive survey of known processes, and recently this summary was brought up to date by Fry [3]. Among the various methods, Lagrange [10] was one of the first to use continued fractions in the solution. On the other hand, at Bell Telephone Laboratories, the solution of the problem has been reduced to a mechanical one by means of the isograph [1], a machine which solves real polynomials up to the tenth degree.

The method described in this paper is similar to that of G. R. Stibitz [3], who divides the complex plane into horizontal and vertical strips, and to that of F. L. Hitchcock [5], who also uses successive approximation and the greatest common divisor process, but entirely by means of rectangular coordinates. Hitchcock's process consists of Horner's method to approximate the real part of the zero and the euclidean algorithm for the greatest common divisor to find the imaginary part. However, the computational routine is relatively complicated compared with the simple processes in the method given below. This simplification is partly due to the formulas given in $\$ 5$ for the continued fraction expansion of a rational function.

2. The method. (i) The separation of the zeros into annular rings. After bounds for the moduli of the zeros of a polynomial have been obtained (see §6), the next step in the computation is the separation of the zeros into different annular rings. In this way, closer bounds for the moduli of the individual zeros are found.

Presented to the Society, April 27, 1946; received by the editors March 20, 1946.

1 Numbers in brackers refer to the bibliography at the end of the paper. 
Let

$$
P(z)=A_{0} z^{n}+A_{1} z^{n-1}+A_{2} z^{n-2}+\cdots+A_{n}
$$

be a polynomial with complex coefficients. By the substitution

$$
z=r \frac{w-1}{w+1}, \quad r>0,
$$

we obtain

$$
\begin{aligned}
P_{r}(w)= & (w+1)^{n} P\left(r \frac{w-1}{w+1}\right) \\
= & \left(p_{0}+i q_{0}\right) w^{n}+\left(p_{1}+i q_{1}\right) w^{n-1}+\left(p_{2}+i q_{2}\right) w^{n-2}+\cdots \\
& +\left(p_{n}+i q_{n}\right) .
\end{aligned}
$$

We form

$$
\begin{aligned}
Q_{r}(w) & =\frac{P_{r}(w) \pm \bar{P}_{r}(-w)}{2} \\
& =i q_{0} w^{n}+p_{1} w^{n-1}+i q_{2} w^{n-2}+p_{3} w^{n-3}+\cdots .
\end{aligned}
$$

Here the positive or negative sign is used according as the degree of $P_{r}(w)$ is odd or even, respectively, and $\bar{P}_{r}(w)$ is obtained from $P_{r}(w)$ by the replacement of all its coefficients by their complex conjugates.

To obtain an algorithm for determining the number of zeros of $P(z)$ interior and exterior to $|z|=r$, we reformulate Theorem 4.1 of [2]: If $P_{r}(w)$ and $Q_{r}(w)$ are of the form (2.3) and (2.4), respectively, and there exists a J-fraction expansion

$$
\begin{aligned}
& \frac{Q_{r}(w)}{P_{r}(w)-Q_{r}(w)}=\frac{1}{c_{1} w+k_{1}}+\frac{1}{c_{2} w+k_{2}}+\cdots+\frac{1}{c_{n} w+k_{n}}, \\
& c_{p} \neq 0 \text {, }
\end{aligned}
$$

where the $c_{p}$ are real and the $k_{p}$ are pure imaginary or zero, then $P_{r}(w)$ has $k$ zeros in $R(w)>0$ and $(n-k)$ zeros in $R(w)<0$ if $k$ of the coefficients $c_{p}$ are negative and the remaining $(n-k)$ are positive. The expansion (2.5) exists only if $q_{0}=0$. We may prove in the same way a similar theorem for the case where $q_{0} \neq 0$. The $J$-fraction expansion is of the form

$$
\begin{aligned}
& \frac{Q_{r}(w)}{P_{r}(w)-Q_{r}(w)} \\
& \quad=\frac{1}{k_{0}}+\frac{1}{c_{1} w+k_{1}}+\frac{1}{c_{2} w+k_{2}}+\cdots+\frac{1}{c_{n} w+k_{n}}, \quad k_{0} \neq 0 .
\end{aligned}
$$


Now, by the transformation (2.2), the interior of $|z|=r$ is mapped onto $R(w)>0$, the exterior of $|z|=r$ onto $R(w)<0$, and $|z|=r$ onto $R(w)=0$. We then have the following application of the above theorem:

THEOREM 2.1. If either the expansion (2.5) or (2.6) exists, the polynomial $P(z)$ (2.1) has $k$ zeros within $|z|=r$ and $(n-k)$ outside if $k$ of the coefficients $c_{p}$ are negative and the remaining $(n-k)$ are positive.

By immediate extension of this theorem, we obtain the following corollary:

THEOREM 2.2. The number of zeros of $P(z)$ in the annular ring $r_{2}>r>r_{1}$ is equal to $k\left(r_{2}\right)-k\left(r_{1}\right)$, where $k\left(r_{2}\right)$ and $k\left(r_{1}\right)$ denote the number of negative coefficients $c_{p}$ for $r=r_{2}$ and $r=r_{1}$, respectively.

In order to eliminate computation with complex numbers, we form, as in [2],

$$
P^{\prime}(w)=i^{n} P_{r}(-i w)=U(w)+i V(w),
$$

where

$$
\begin{aligned}
& U(w)=p_{0} w^{n}-q_{1} w^{n-1}-p_{2} w^{n-2}+q_{3} w^{n-3}+p_{4} w^{n-4}-\cdots, \\
& V(w)=q_{0} w^{n}+p_{1} w^{n-1}-q_{2} w^{n-2}-p_{3} w^{n-3}+q_{4} w^{n-4}+\cdots .
\end{aligned}
$$

Then, if $q_{0}=0$, we have in general

$$
\begin{aligned}
\frac{V(w)}{U(w)} & =\frac{1}{d_{1} w+m_{1}}+\frac{1}{d_{2} w+m_{2}}+\cdots+\frac{1}{d_{n} w+m_{n}} \\
& =\frac{1}{c_{1} w+i k_{1}}-\frac{1}{c_{2} w+i k_{2}}-\cdots-\frac{1}{c_{n} w+i k_{n}}
\end{aligned}
$$

where

$$
\text { (2.10) } \quad c_{p}=(-1)^{p+1} d_{p}, \quad i k_{p}=(-1)^{p+1} m_{p}, \quad p=1,2, \cdots, n \text {. }
$$

If $q_{0} \neq 0$, the $J$-fraction expansion in general is of the form

$$
\begin{aligned}
\frac{V(w)}{U(w)} & =\frac{1}{m_{0}}+\frac{1}{d_{1} w+m_{1}}+\frac{1}{d_{2} w+m_{2}}+\cdots+\frac{1}{d_{n} w+m_{n}} \\
& =\frac{1}{i k_{0}}-\frac{1}{c_{1} w+i k_{1}}-\frac{1}{c_{2} w+i k_{2}}-\cdots-\frac{1}{c_{n} w+i k_{n}}
\end{aligned}
$$

where

(2.12) $\quad c_{p}=(-1)^{p} d_{p}, i k_{0}=m_{0}, i k_{p}=(-1)^{p} m_{p}, \quad p=1,2, \cdots, n$. 
In $\$ 5$ explicit formulas are given for finding the expansions (2.9) and (2.11).

(ii) Computation of the zeros of $P(z)$ of known modulus. From the mapping (2.2) we see that

$$
z^{\prime}=r \frac{w^{\prime}-1}{w^{\prime}+1}
$$

is a zero of $P(z)$ of modulus $r$ if $w^{\prime}$ is a pure imaginary zero of $P\left(r\left(w^{\prime}-1\right) /\left(w^{\prime}+1\right)\right)$, where 0 and $\infty$ are included. But the pure imaginary zeros of $(w+1)^{n} P(r(w-1) /(w+1))$ are precisely the pure imaginary zeros of $D_{r}(w)$ which is the greatest common divisor of $P_{r}(w)$ and $Q_{r}(w)$. We may obtain $D_{r}(w)$ by the euclidean algorithm and therefore the zeros of $P(z)$ by (2.13). As in (i), we may reduce our problem to that of computation with real numbers by finding $D_{r}^{\prime}(w)$, the greatest common divisor of $U(w)$ and $V(w)$, and then set $D_{r}(w)$ $=D_{r}^{\prime}(i w)$.

In particular, if $-r$ is a zero of $P(z)$, then $w=0$ is a common zero of $P_{r}(w)$ and $Q_{r}(w)$; if $+r$ is a zero of $P(z)$, then $w=\infty$ is a common zero of $P_{r}(w)$ and $Q_{r}(w)$.

(iii) Computation of the zeros of $P(z)$ if the moduli are not known. By successively varying the values of $r$ such that the last remainder approaches zero in the euclidean algorithm and such that simultaneously there is a change in the number of positive and negative signs of the $c_{p}$ in the continued fraction expansions (2.5) or (2.6), we may approximate as closely as we wish the value of $D_{r}(w)$ from the last nonzero remainder. From the zeros $w^{\prime}$ of this approximation to $D_{r}(w)$ we may obtain the zeros of $P(z)$, namely $z^{\prime}=r\left(w^{\prime}-1\right) /\left(w^{\prime}+1\right)$, as closely as desired. A simple method to obtain a correction in the value of $r$ and also in the value of $z^{\prime}$ is by interpolation between the values of the remainders corresponding to a change in the number of positive and negative signs of the $c_{p}$. The computation may be conveniently arranged in tabular form as shown in $\$ 5$.

3. Cases where the $J$-fraction expansion does not exist. If, in the euclidean algorithm for the greatest common divisor of $Q_{r}(w)$ and $P_{r}(w)-Q_{r}(w)$ (see formulas (5.1) or (5.3)), a zero remainder is obtained before $n$ steps have been carried out, then we find the zeros of the first nonzero remainder $D_{r}(w)$.

If, however, one of the $c_{p}, p \neq n$, is zero and yet the remainder corresponding to this $c_{p}$ is not zero, we again cannot expand $Q_{r}(w) /\left(P_{r}(w)\right.$ $\left.-Q_{r}(w)\right)$ into a $J$-fraction. This case occurs when certain determinants vanish (cf. [2]). We may then proceed as follows: If, for $r$ 
near $r_{1}$, the $J$-fraction expansion shows a change in the number of positive and negative signs of the $c_{p}$ and for $r=r_{1}$ one of the $c_{p}, p \neq n$, vanishes, then $r=r_{1}$ is the modulus of the desired zero. However, the corresponding value of $w$ cannot always be found from the next to the last remainder. We then make a transformation $z=z^{\prime}+k$, where $k$ is a constant, and find in the same way the modulus $r=r_{2}$ of the same zero of $P\left(z^{\prime}\right)$. From the triangle thus formed, we compute $z=x+i y$, the desired zero of $P(z)$.

4. Computation of the zeros of polynomials with real coefficients. If the coefficients of $P(z)$ are real, the continued fraction expansion (2.9) may be computed from table (5.3). However, it is advisable to first determine whether the zeros of $P(z)$ are all real by the following theorem (cf., for example, Grommer [4, pp. 123-125]).

THEOREM 4.1. If

$$
P(z)=A_{0} z^{n}+A_{1} z^{n-1}+A_{2} z^{n-2}+\cdots+A_{n}, \quad A_{0}>0,
$$

has real coefficients, all its zeros are real and different if and only if

$$
\frac{P^{\prime}(z)}{P(z)}=\frac{1}{r_{1} z+s_{1}}+\frac{1}{r_{2} z+s_{2}}+\cdots+\frac{1}{r_{n} z+s_{n}},
$$

where the $r_{p}$ and $s_{p}$ are real and the $r_{p}$ are positive or negative according as $p$ is odd or even, respectively.

The proof easily follows by an application of Sturm sequences.

That $P(z)$ has no multiple zeros is not an essential restriction, for, in the case of multiple zeros, $P(z)$ and $P^{\prime}(z)$ have common factors which may first be removed by the euclidean algorithm.

The following theorem of Van Vleck [16] gives a method to ascertain whether a real polynomial contains the maximum number of imaginary zeros.

ThEOREM 4.2. If the $A_{p}$ are real and the terms of the sequence

$$
\begin{gathered}
A_{0},\left|\begin{array}{ll}
A_{0} & A_{1} \\
A_{1} & A_{2}
\end{array}\right|,\left|\begin{array}{lll}
A_{0} & A_{1} & A_{2} \\
A_{1} & A_{2} & A_{3} \\
A_{2} & A_{3} & A_{4}
\end{array}\right|, \cdots, \\
\Delta_{n}=\left|\begin{array}{ccccc}
A_{0} & A_{1} & A_{2} & \cdots & A_{n} \\
A_{1} & A_{2} & A_{3} & \cdots & A_{n+1} \\
\cdot & \cdot & \cdots & \cdots & \cdots \\
A_{n} & A_{n+1} & A_{n+2} & \cdots & A_{2 n}
\end{array}\right|
\end{gathered}
$$


are positive, then all the zeros of

$$
P_{2 n}(z)=A_{0}+A_{1} z+A_{2} z^{2}+\cdots+A_{2 n} z^{2 n}
$$

are imaginary, and all but one of the zeros of

$$
P_{2 n+1}(z)=A_{0}+A_{1} z+A_{2} z^{2}+\cdots+A_{2 n+1} z^{2 n+1} .
$$

5. Formulas for the expansion of a rational function into a $J$-fraction. In [2] we presented an algorithm for the expansion of a rational function

$$
\frac{f_{1}}{f_{0}}=\frac{\alpha_{11} w^{n-1}+\alpha_{12} w^{n-2}+\cdots+\alpha_{1 n}}{\alpha_{00} w^{n}+\alpha_{01} w^{n-1}+\cdots+\alpha_{0 n}}
$$

into a $J$-fraction of the form (2.5) (see table (2.4), p. 146), and we showed that the expansion (2.5) exists if and only if $\alpha_{00} \neq 0$ and certain determinants of odd order are different from zero (formulas (2.8)).

In a similar way, it can be shown that

$$
\frac{F_{1}}{F_{0}}=\frac{\alpha_{00} w^{n}+\alpha_{01} w^{n-1}+\cdots+\alpha_{0 n}}{\beta_{00} w^{n}+\beta_{01} w^{n-1}+\cdots+\beta_{0 n}}
$$

may be expanded into a $J$-fraction of the form (2.6) by the following formulas:

$$
\begin{aligned}
& \begin{array}{llll}
\alpha_{00}=q_{0}, & \alpha_{01}=p_{1}, & \alpha_{02}=-q_{2}, & \ldots, \\
\beta_{00}=p_{0}, & \beta_{01}=-q_{1}, & \beta_{02}=-p_{2}, & \ldots, \\
\alpha_{11}=\frac{\alpha_{00} \beta_{01}-\beta_{00} \alpha_{01}}{\alpha_{00}}, & \alpha_{12}=\frac{\alpha_{00} \beta_{02}-\beta_{00} \alpha_{02}}{\alpha_{00}}, & \alpha_{13}=\frac{\alpha_{00} \beta_{03}-\beta_{00} \alpha_{03}}{\alpha_{00}}, \ldots,
\end{array} \\
& \beta_{11}=\frac{\alpha_{11} \alpha_{01}-\alpha_{00} \alpha_{12}}{\alpha_{11}}, \beta_{12}=\frac{\alpha_{11} \alpha_{02}-\alpha_{00} \alpha_{13}}{\alpha_{11}}, \beta_{13}=\frac{\alpha_{11} \alpha_{03}-\alpha_{00} \alpha_{14}}{\alpha_{11}}, \ldots, \\
& \alpha_{22}=\frac{\alpha_{11} \beta_{12}-\beta_{11} \alpha_{12}}{\alpha_{11}}, \alpha_{23}=\frac{\alpha_{11} \beta_{13}-\beta_{11} \alpha_{13}}{\alpha_{11}}, \alpha_{24}=\frac{\alpha_{11} \beta_{14}-\beta_{11} \alpha_{14}}{\alpha_{11}}, \ldots, \\
& \beta_{22}=\frac{\alpha_{22} \alpha_{12}-\alpha_{11} \alpha_{23}}{\alpha_{22}}, \beta_{23}=\frac{\alpha_{22} \alpha_{13}-\alpha_{11} \alpha_{24}}{\alpha_{22}}, \beta_{24}=\frac{\alpha_{22} \alpha_{14}-\alpha_{11} \alpha_{25}}{\alpha_{22}}, \ldots, \\
& d_{p}=\frac{\alpha_{p-1, p-1}}{\alpha_{p, p}}, \quad m_{0}=\frac{\beta_{00}}{\alpha_{00}}, \quad m_{p}=\frac{\beta_{p, p}}{\alpha_{p, p}}, \quad p=1,2, \cdots, n .
\end{aligned}
$$

The expansion (2.6) exists if and only if $D_{0}=\alpha_{00} \neq 0$ and the determinants $D_{p}, p=1,2, \cdots, n$, formed from the first $n$ principal minors of even order in the array 


\begin{tabular}{ll|ll|ll|ll}
$\alpha_{00}$ & $\alpha_{01}$ & $\alpha_{02}$ & $\alpha_{03}$ & $\alpha_{04}$ & $\alpha_{05}$ & $\alpha_{06}$ & $\ldots$ \\
$\beta_{00}$ & $\beta_{01}$ & $\beta_{02}$ & $\beta_{03}$ & $\beta_{04}$ & $\beta_{05}$ & $\beta_{06}$ & $\ldots$ \\
\cline { 1 - 5 } 0 & $\alpha_{00}$ & $\alpha_{01}$ & $\alpha_{02}$ & $\alpha_{03}$ & $\alpha_{04}$ & $\alpha_{05}$ & $\ldots$ \\
0 & $\beta_{00}$ & $\beta_{01}$ & $\beta_{02}$ & $\beta_{03}$ & $\beta_{04}$ & $\beta_{05}$ & $\ldots$ \\
\hline 0 & 0 & $\alpha_{00}$ & $\alpha_{01}$ & $\alpha_{02}$ & $\alpha_{03}$ & $\alpha_{04}$ & $\ldots$ \\
0 & 0 & $\beta_{00}$ & $\beta_{01}$ & $\beta_{02}$ & $\beta_{03}$ & $\beta_{04}$ & $\ldots$ \\
\hline 0 & 0 & 0 & $\alpha_{00}$ & $\alpha_{01}$ & $\alpha_{02}$ & $\alpha_{03}$ & $\ldots$
\end{tabular}

are different from zero, where $\alpha_{0 p}=\beta_{0 p}=0$ if $p>n$.

As a special case of table (2.4) of [2], the rational function

$$
\frac{\phi_{1}}{\phi_{0}}=\frac{\alpha_{11} w^{n-1}+\alpha_{12} w^{n-3}+\alpha_{13} w^{n-5}+\cdots}{\alpha_{00} w^{n}+\alpha_{01} w^{n-2}+\alpha_{02} w^{n-4}+\cdots}
$$

may be expanded into a $J$-fraction of the form (2.5) by the formulas

$$
\begin{aligned}
& \alpha_{00}, \quad \alpha_{01}, \quad \alpha_{02}, \quad \ldots \\
& \alpha_{11}, \quad \alpha_{12}, \quad \alpha_{13} \\
& \alpha_{22}=\frac{\alpha_{11} \alpha_{01}-\alpha_{00} \alpha_{12}}{\alpha_{11}}, \alpha_{23}=\frac{\alpha_{11} \alpha_{02}-\alpha_{00} \alpha_{13}}{\alpha_{11}}, \alpha_{24}=\frac{\alpha_{11} \alpha_{03}-\alpha_{00} \alpha_{14}}{\alpha_{11}}, \ldots, \\
& (5.3)=\frac{\alpha_{22} \alpha_{12}-\alpha_{11} \alpha_{23}}{\alpha_{33}}, \quad \alpha_{34}=\frac{\alpha_{22} \alpha_{13}-\alpha_{11} \alpha_{24}}{\alpha_{22}}, \alpha_{35}=\frac{\alpha_{22} \alpha_{14}-\alpha_{11} \alpha_{25}}{\alpha_{22}}, \ldots, \\
& d_{p}=\frac{\alpha_{p-1, p-1}}{\alpha_{p, p}}, m_{p} \equiv 0, \quad \quad p=1,2,3, \cdots,
\end{aligned}
$$

provided $\alpha_{00} \neq 0$ and certain determinants of odd and even order are different from zero (the principal minors in the array (2.8) of [2]).

6. Upper and lower bounds for the moduli of the zeros of $P(z)$. In [2] a method was given for finding bounds for the moduli of the zeros of a polynomial. We apply the principles of this method to obtain better bounds by means of Cassinian ovals.

Let

(6.1) $P(z)=z^{n}+\left(a_{1}+i b_{1}\right) z^{n-1}+\left(a_{2}+i b_{2}\right) z^{n-2}+\cdots+\left(a_{n}+i b_{n}\right)$, where the coefficient of $z^{n}$ is taken to be unity since the polynomial may always be reduced to this form. The expansion (2.5) will in general exist and, after simple transformations, it may be written in the form 


$$
\frac{Q(z)}{P(z)}=\frac{\left(c_{1} z+k_{1}+1\right)^{-1}}{1}+\frac{h_{1}}{1}+\frac{h_{2}}{1}+\cdots+\frac{h_{n-1}}{1},
$$

where

$$
\begin{aligned}
h_{1}=\frac{1}{\left(c_{1} z+k_{1}+1\right)\left(c_{2} z+k_{2}\right)}, \quad h_{2} & =\frac{1}{\left(c_{2} z+k_{2}\right)\left(c_{3} z+k_{3}\right)}, \\
\cdots, & h_{n-1}=\frac{1}{\left(c_{n-1} z+k_{n-1}\right)\left(c_{n} z+k_{n}\right)} .
\end{aligned}
$$

If $g_{1}, g_{2}, \cdots, g_{n-1}$ are numbers such that $0<g_{p}<1, p=1,2, \cdots$, $n-1$, then $P(z) \neq 0$ if $z$ satisfies the inequalities $\left|h_{1}\right| \leqq g_{1},\left|h_{2}\right|$ $\leqq\left(1-g_{1}\right) g_{2},\left|h_{3}\right| \leqq\left(1-g_{2}\right) g_{3}, \cdots,\left|h_{n-1}\right| \leqq\left(1-g_{n-2}\right) g_{n-1}$. Hence each zero of $P(z)$ must lie within at least one of the Cassinian ovals

$$
\begin{aligned}
\left|\left(c_{1} z+k_{1}+1\right)\left(c_{2} z+k_{2}\right)\right| & =\frac{1}{g_{1}} \\
\left|\left(c_{2} z+k_{2}\right)\left(c_{3} z+k_{3}\right)\right| & =\frac{1}{\left(1-g_{1}\right) g_{2}}, \cdots, \\
\left|\left(c_{n-1} z+k_{n-1}\right)\left(c_{n} z+k_{n}\right)\right| & =\frac{1}{\left(1-g_{n-2}\right) g_{n-1}} .
\end{aligned}
$$

Then an upper bound for the moduli of the zeros is obtained from the point of maximum modulus of all these curves. The point with largest modulus on each oval is on the line joining its two fixed points.

The methods of this paper may be applied to advantage to a polynomial whose zeros are relatively close together, as, for example, in the case of the polynomial (cf. Hitchcock [5])

(6.5) $P(z)=z^{3}+(.4-i) z^{2}+(6.67-.2 i) z+(.0667-6.67 i)$.

An upper bound of 2.866 for the moduli of the zeros is found by the use of Cassinian ovals with $g_{1}=56 / 59, g_{2}=160 / 168$, and a lower bound of .47 with $g_{1}=85 / 175, g_{2}=175 / 204$. By the methods of $\$ 2$, the moduli are actually found to be 2.590596, 2.580912, .997642, and the corresponding zeros are $-.254757+2.578039 i,-.168394$ $-2.575413 i, .023149+.997373 i$.

\section{BIBLIOGRAPHY}

1. R. L. Dietzold, The isograph-a mechanical root-finder, Bell Laboratories Record vol. 16 (1937) pp. 130-134.

2. Evelyn Frank, On the zeros of polynomials with complex coefficients, Bull. Amer. Math. Soc. vol. 52 (1946) pp. 144-158. 
3. Thornton C. Fry, Some numerical methods for locating roots of polynomials, Quarterly of Applied Mathematics vol. 3 (1945) pp. 89-105.

4. J. Grommer, Ganze transzendente Funktionen mit lauter reellen Nullstellen, J. Reine Angew. Math. vol. 144 (1914) pp. 114-165.

5. Frank L. Hitchcock, Algebraic equations with complex coefficients, Journal of Mathematics and Physics (Massachusetts Institute of Technology) vol. 18 (1939) pp. 202-210.

6. A. Hurwitz, Über die Bedingungen, unter welchen eine Gleichung nur Wurzeln mit negativen reellen Teilen besitzt, Math. Ann. vol. 46 (1895) pp. 273-284 (Werke, vol. 2, pp. 533-545).

7. - Über die Nullstellen der Bessel'schen Funktion, Math. Ann. vol. 33 (1889) pp. 246-266 (Werke, vol. 1, pp. 266-286).

8. - Über einen Satz des Herrn. Kakeya, Tôhoku Math, J. vol. 4 (1913) pp. 89-93 (Werke, vol. 2, pp. 627-631).

9. A. J. Kempner, On the complex roots of algebraic equations, Bull. Amer. Math. Soc. vol. 41 (1935) pp. 809-843.

10. J. L. Lagrange, Traité de la resolution des équations numériques de tous les Degres, Oeuvres, vol. 5, Coucier, Paris, 1808.

11. O. Perron, Die Lehre von den Kettenbriuchen, Teubner, Leipzig, 1929.

12. C. Runge, Separation und Approximation der Wurzeln, Encyklopädie der Mathematischen Wissenschaften I, 1, Teubner, Leipzig, 1898, pp. 404-448.

13. - - Praxis der Gleichungen, Sammlung Schubert, no. 14, Göschen, Leipzig, 1900.

14. C. Runge and H. König, Vorlesungen ibber numerisches Rechnen, Die Grundlehren der Mathematischen Wissenschaften, vol. 11, Springer, Berlin, 1924.

15. I. Schur, Über Potenzreihen, die im Innern des Einheitskreises beschränt sind, J. Reine Angew. Math. vol. 147 (1917) pp. 205-232; vol. 148 (1918) pp. 122-145.

16. E. B. Van Vleck, $A$ sufficient condition for the maximum number of imaginary roots of an equation of the nth degree, Ann. of Math. (2) vol. 4 (1903) pp. 191-192.

NORTHWESTERN UNIVERSITY 